\title{
Exploring a brightness-drag illusion
}

\section{Tina Habota, Mark Chappell}

Department of Psychology (MG) and Applied Cognitive Neuroscience Unit, Griffith Health Institute, Griffith University, Nathan, 4111 QLD, Australia; e-mail: m.chappell@griffith.edu.au

Received 2 July 2010, in revised form 5 November 2010

\begin{abstract}
A sudden luminance increment on a moving stimulus was perceived significantly along the trajectory, in the direction of motion, from its displayed position. A nonlinear relationship with stimulus speed, for a Fröhlich-like illusion, but not for the luminance-increment illusion, challenges certain models of spatial mislocalisation and argues for different processes underlying the two illusions.
\end{abstract}

Moving stimuli often transit between regions of different illumination but it may be important that the visual system's computation of position is not dramatically disrupted by this, for example at a late-afternoon game at Wimbledon. There has been considerable work on the perception of the position and/or timing of an (ecologically implausible) colour change from red to green on moving stimuli (eg Kreegipuu and Allik 2004; Moutoussis and Zeki 1997). However, as these colours are processed in an opponent fashion, it is likely that a complicated dynamic accompanies an abrupt change from one to the other, in line with Nishida and Johnston's (2002) finding that very different illusions result when there are repeated changes between red and green and back again, as opposed to a single change. A change in luminance not only corresponds to likely changes in the environment - it is also likely to engage fewer and simpler processes, thus leading to more easily interpretable results. However, we are aware of only one study of the perceived position of a simple change in luminance on a moving stimulus (Kerzel 2003). In that study decrements only have been examined, and the position of the decrement was found to be misperceived in the direction of motion.

Our participants compared the perceived position of a sudden increase in luminance $\left(\sim 3 \mathrm{~cd} \mathrm{~m}^{-2}\right.$ to $\sim 115 \mathrm{~cd} \mathrm{~m}^{-2}$, background $\sim 1 \mathrm{~cd} \mathrm{~m}^{-2}$ ) on a moving stimulus (at 1.35, 4.35 , and $12 \mathrm{deg} \mathrm{s}^{-1}$ ) with that of a landmark stimulus which appeared just before the increment change, ${ }^{(1)}$ and remained on for the remainder of the trial. For comparison with this increment trajectory, our participants also compared the point of appearance of a moving stimulus with the landmark (both appearing simultaneously) - an onset trajectory. Research with the Fröhlich illusion (reviewed by Whitney 2002) suggested this point would be systematically misperceived in the direction of motion.

As indicated by the $95 \%$ confidence intervals in figure 1 , for individual participants the increment illusion was significant at all speeds for at least eight out of the twelve participants. Also revealed is a general tendency for participants' data to depart from linearity, or to exhibit a more pronounced compressive nonlinearity (where the graph with speed 'curves over', but is still monotonically increasing), with the onset than with the increment trajectory. The $95 \%$ confidence intervals on the averages demonstrate that across participants all speeds produced a significant illusion with both trajectory types. As the literature did not provide much guidance as to the form of relationship we should expect (see below), we planned to separately test our increment and onset trajectories for linearity, as a function of speed. Using a metric (135, 453, and 1200),

- Author to whom all correspondence should be addressed.

(1) Owing to an oversight, one frame $(16.67 \mathrm{~ms})$ before the increment change. 

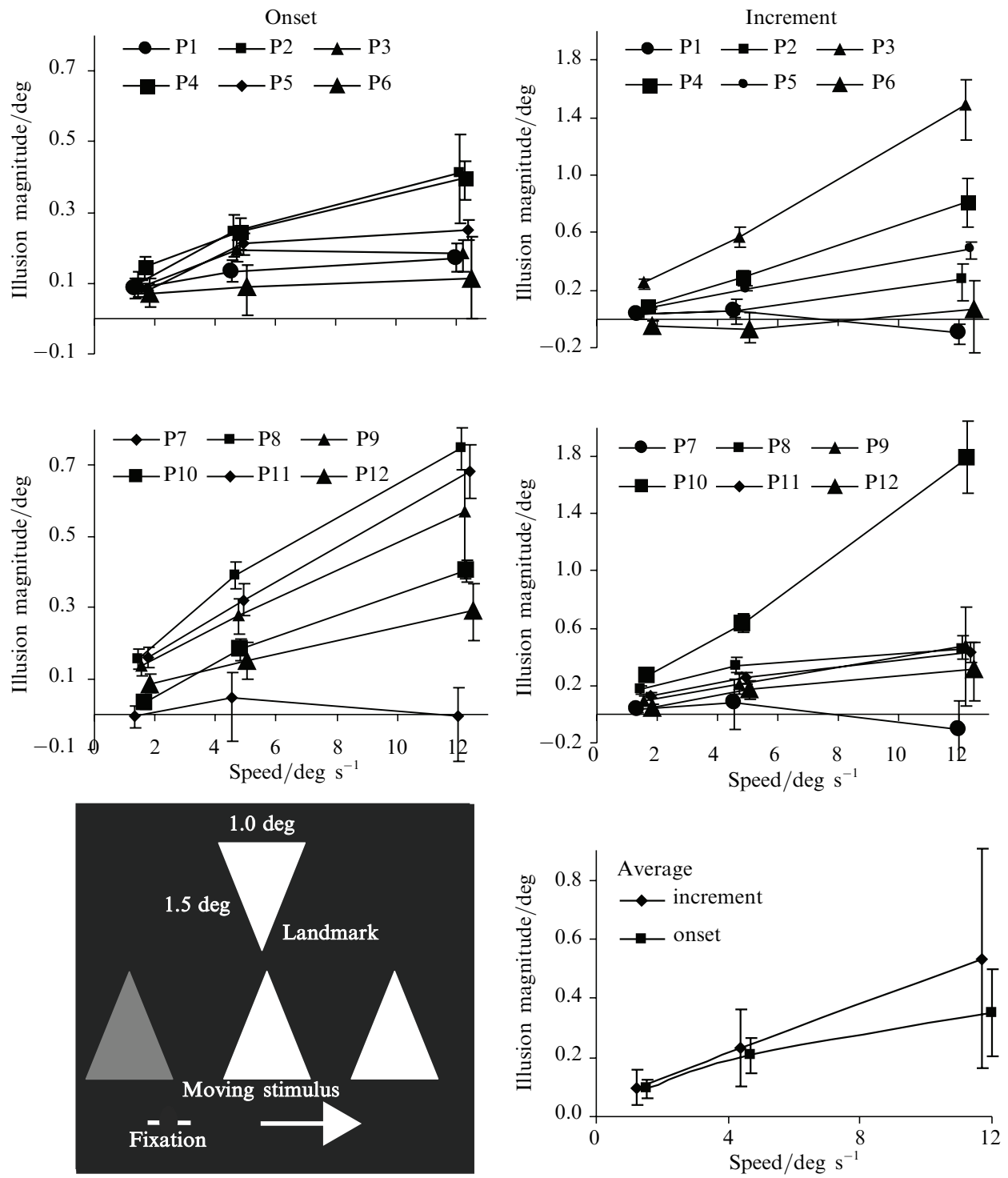

Figure 1. Stimuli, individual (method of fixed stimuli and logistic regression on 144 trials/condition/ participant) and average (bottom right panel) results for 12 participants (P1 - P12). Error bars are 95\% confidence intervals. Note the different scale for the onset (left) and increment (right) trajectories, for individual-participant data.

which accounted for the unequal spacing between the speeds, for the onset trajectory type an ANOVA revealed a significant linear effect $\left(F_{1,11}=19.72, p=0.001, \eta_{\mathrm{p}}^{2}=0.64\right)$ as well as a significant quadratic effect $\left(F_{1,11}=21.62, p=0.001, \eta_{\mathrm{p}}^{2}=0.66\right)$. For the increment trajectory type, there was a significant linear effect $\left(F_{1,11}=8.61, p=0.014\right.$, $\left.\eta_{\mathrm{p}}^{2}=0.44\right)$, but no quadratic effect $\left(p=0.79, \eta_{\mathrm{p}}^{2}=0.007\right)$.

The position of an increment in luminance was systematically misperceived in the direction of motion, complementing Kerzel's (2003) similar finding with a luminance decrement. As an explanation of this brightness-drag illusion we will focus on what we call 'differential latency for attribute'. Following Moutoussis and Zeki (1997) and Kerzel (2003), we phrase it thus: by the time the change in luminance has been processed, the processing of the moving object's position supports its perception further along 
its trajectory. When the luminance change is available to perception it is attached, or bound, to the currently perceived position of the moving object. Provided the differential latency between processing of position and luminance is constant as a function of speed, our linear dependence of the increment illusion on speed is accounted for.

While the comparison stimulus with our onset trajectory was not constantly visible for the duration of the trial, as we believe it should be for a pure Fröhlich paradigm, we would expect our paradigm to yield very similar results to that one. Some studies of the Fröhlich illusion have found an approximately linear relationship (eg experiments 1, 3, and 5 of Kerzel and Müsseler 2002), whereas others have found a compressively nonlinear relationship with speed (eg Müsseler and Aschersleben 1998; for review see also Kerzel and Gegenfurtner 2004; Kreegipuu and Allik 2003).

Of course, it would be desirable to replicate our finding with more levels of speed. Should the nonlinear relationship prove to be a reliable finding with the onset trajectory, then differential latency for luminance change would need to be supplemented, or replaced, with other nonlinear (with speed) processes, as would the simplest forms of temporal integration (Lappe and Krekelberg 1998) and spatial projection (Nijhawan 1994; Eagleman and Sejnowski 2007) accounts. That different processes are involved in the two trajectories is supported by the fact that correlations computed for each of the three speeds, between illusion magnitudes with the different illusions, were all nonsignificant $(p \mathrm{~s}>0.25){ }^{(2)}$ and indeed it would be surprising if the appearance of a new moving stimulus did not entail more processes (Yantis 1996).

Players do misperceive the position of luminance changes on tennis balls, but whether this affects their perception of the position of the ball remains an Open question.

Acknowledgments. We would like to thank the Applied Cognitive Neuroscience Unit for support, and David Hardwick for help with graphics. This work was the basis of an Honours Thesis project for the first author.

\section{References}

Eagleman D M, Sejnowski T J, 2007 "Motion signals bias localization judgments: A unified explanation for the flash-lag, flash-drag, flash-jump, and Fröhlich illusions" Journal of Vision 7(4):3, $1-12$

Kerzel D, 2003 "Asynchronous perception of motion and luminance change" Psychological Research $67233-239$

Kerzel D, Gegenfurtner K R, 2004 "Spatial distortions and processing latencies in the onset repulsion and Fröhlich effects" Vision Research $44577-590$

Kerzel D, Müsseler J, 2002 "Effects of stimulus material on the Fröhlich illusion" Vision Research $42181-189$

Kreegipuu K, Allik J, 2003 "Perceived onset time and position of a moving stimulus" Vision Research $431625-1635$

Kreegipuu K, Allik J, 2004 "Confusion of space and time in the flash-lag effect" Perception 33293 - 306

Lappe M, Krekelberg B, 1998 "The position of moving objects" Perception 27 1437-1449

Müsseler J, Aschersleben G, 1998 "Localizing the first position of a moving stimulus: The Fröhlich effect and an attention-shifting explanation" Perception \& Psychophysics $60683-695$

Moutoussis K, Zeki S, 1997 "A direct demonstration of perceptual asynchrony in vision" Proceedings of the Royal Society of London, Series B 264393 - 399

Nijhawan R, 1994 "Motion extrapolation in catching" Nature 370 256-257

Nishida S, Johnston A, 2002 "Marker correspondence, not processing latency, determines temporal binding of visual attributes" Current Biology $12359-368$

Whitney D, 2002 "The influence of visual motion on perceived position" Trends in Cognitive Sciences $6211-216$

Yantis S, 1996 "Attentional capture in vision", in Converging Operations in the Study of Visual Selective Attention Eds A F Kramer, M G H Coles, G D Logan (Washington, DC: APA Press) pp 45-76

(2) Suggested by an anonymous reviewer. 


\section{PERCEPTION}

VOLUME 402011

www.perceptionweb.com

Conditions of use. This article may be downloaded from the Perception website for personal research by members of subscribing organisations. Authors are entitled to distribute their own article (in printed form or by e-mail) to up to 50 people. This PDF may not be placed on any website (or other online distribution system) without permission of the publisher. 